\title{
Towards Understanding Egyptian Arabic Dialogues
}

\author{
AbdeIRahim A. Elmadany \\ Department of Science, Institute of \\ Statistical Studies and Research \\ (ISSR), Cairo University
}

\author{
Sherif M. Abdou \\ Department of Information \\ Technology, Faculty of Computers \\ and Information, Cairo University
}

\author{
Mervat Gheith \\ Department of Science, Institute of \\ Statistical Studies and Research \\ (ISSR), Cairo University
}

\begin{abstract}
Labelling of user's utterances to understanding his attends which called Dialogue Act (DA) classification, it is considered the key player for dialogue language understanding layer in automatic dialogue systems. In this paper, we proposed a novel approach to user's utterances labeling for Egyptian spontaneous dialogues and Instant Messages using Machine Learning (ML) approach without relying on any special lexicons, cues, or rules. Due to the lack of Egyptian dialect dialogue corpus, the system evaluated by multi-genre corpus includes 4725 utterances for three domains, which are collected and annotated manually from Egyptian call-centers. The system achieves $F_{1}$ scores of $70.36 \%$ overall domains.
\end{abstract}

\section{General Terms}

Natural Language Processing, Machine Learning, Support Vector Machine.

\section{Keywords}

Dialogue Act Classification, Arabic Dialogue Understanding, Egyptian Arabic Dialect, Arabic Instant Messages.

\section{INTRODUCTION}

Knowing the user needs is considered the most important part and the difficult part to build a better human-computer system; which called language understating or somewhere Dialogue Acts (DAs) classification. DAs classification task is labelling the speaker's intention in producing a particular utterance with short words; the DAs terminology is approximately the equivalent of the speech act of Searle (1969) and DAs is different based on dialogue systems domains [1]. Hence, within the field of computational linguistics closely linked to the development and deployment of spoken language dialogue systems, has focused on the some of the more conversational roles such acts can perform. The research on DAs has increased since 1999, after spoken dialogue systems became a commercial reality [2].

This paper refers to an utterance as a small unit of speech that corresponds to a single act [3, 4]. In speech research community, utterance definition is a slightly different; it refers to a complete unit of speech bounded by the speaker's silence while, we refer to the complete unit of speech as $a$ turn. Thus, a single turn can be composed of many utterances. Turn and utterance can be the same definition when the turn contains one utterance as defined and used in [5] .

There are two approaches to building a DAs classifier: semantic approach and syntax approach. In semantic approach, segment long turns into utterances task is not important because this approach based on turn semantic labelling using identifying the key sequence that called "conceptual segments "or "cues" from the turn. In syntax approach, turn segmentation into utterances task is important segmentation because this approach based morphological features and linguistic rules.

In this paper, we present an approach to understanding spontaneous dialogues for Arabic namely "YOSR". It is a machine learning approach based on context without relying on text diacritization or lexical cues. Whereas, YOSR depends on a set of features from the annotated data that's included morphological features which have been determined by the Morphological Analysis and Disambiguation of Arabic Tool (MADAMIRA) $^{1}$ [6], and utterances features. YOSR is evaluated by an Arabic dialogue corpus contains spoken dialogues and Instant Messages (IM) for Egyptian Arabic, and results are compared with manually annotated utterances elaborated by experts.

This paper is organized as follows: section 2 present the challenges of Arabic dialogues understanding, section 3 present the background and related works, section 4 present the proposed classifier "YOSR", section 5 present the experimental setup and results; and finally the conclusion and feature works is reported in section 6 .

\section{ARABIC CHALLENGES}

Arabic is one of the six official languages of the United Nations. According to Egyptian Demographic Center, it is the mother tongue of about 300 million people (22 countries). There are about 135.6 million Arabic internet users until $2013^{2}$.

The orientation of writing is from right to left and the Arabic alphabet consists of 28 letters. The Arabic alphabet can be extended to ninety elements by writing additional shapes, marks, and vowels. Most Arabic words are morphologically derived from a list of roots that are tri, quad, or pent-literal. Most of these roots are tri-literal. Arabic words are classified into three main parts of speech, namely nouns, including adjectives and adverbs, verbs, and particles. In formal writing, Arabic sentences are often delimited by commas and periods. Arabic language has two main forms: Standard Arabic and Dialectal Arabic. Standard Arabic includes Classical Arabic (CA) and Modern Standard Arabic (MSA) while Dialectal Arabic includes all forms of currently spoken Arabic in daily life, including online social interaction and it vary among countries and deviate from the Standard Arabic to some extent [7]. There are six dominant dialects, namely; Egyptian, Moroccan, Levantine, Iraqi, Gulf, and Yemeni.

MSA considered as the standard that commonly used in books, newspapers, news broadcast, formal speeches, movie subtitles... etc. Egyptian dialects commonly known as

\footnotetext{
${ }^{1}$ http://nlp.ldeo.columbia.edu/madamira/

http://www.internetworldstats.com/stats $7 . h$ htm
} 
Egyptian colloquial language are the most widely understood Arabic dialects due to a thriving Egyptian television and movie industry, and Egypt's highly influential role in the region for much of the 20th century [8]. Egyptian dialect has several large regional varieties such as Delta and Upper Egypt, but the standard Egyptian Arabic is based on the dialect of the Egyptian capital, which is the most understood by all Egyptians.

Due to the lack of an Egyptian Arabic recognition system, manual transcription of the corpus is then required. Moreover, Understanding spontaneous Arabic dialogues task has several challenges:

- Essential characteristics of spontaneous speech: ellipses, anaphora, hesitations, repetitions, repairs... etc. These are some examples from our corpus:

م A user who does repairs and apologize in his turn: السفر (Alsfr ywm 12 dysmbr Asfh 11 dysmbr, the arrival on 12 sorry 11 December) $)^{3}$.

- A user who repeats the negative answer and produce لا لا انا مش فاتحة : non-necessary information on his turn حساب عندكم وانا مبشتغلس بس جوزي هو اللي بيشتغل $m \$$ fAtHp HsAb Endkm wAnA mb\$tgls bs jwzy hw Ally by $\$ t g l$, No No I don't have an account in your bank and I'm not an employee but my husband is an employee)

- Code Switching: using a dialect words which are derived from foreign languages by code switching between Arabic and other language such as English, France, or Germany. Here an example for user who uses foreign "Egnlish" words in his turn such as نر انز اكثن (trAnzAk\$n, Transaction)

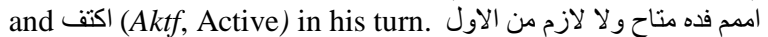
اعمل اي تر انز اكثن علثان ييقي اكتف بدل دورمنت (Ammm fdh mtAH wlA lAzm mn AlAwl AEml Ay trAnzAk\$n El\$An ybqy Aktf $b d l$ dwrmnt, Um this is available or I need to do any transaction to activate the dormant account)

- Deviation: Dialect Arabic words may be having some deviation such as MSA "اريد" (Aryd, want) can be "عايز" (EAyz, want), or "عاوز" (EAwz, want) in Egyptian dialect.

- Ambiguity: Arabic word may be having different means

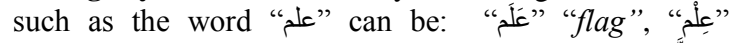

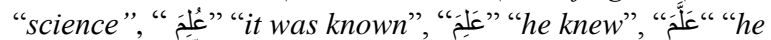
taught" or "عُلَّ" "he was taught". Thus, the ambiguity considers the key problem for Natural Language Understanding/Processing especially on the Arabic language. The word diacritization is very useful to clarify the meaning of words and disambiguate any vague spellings.

- Lack of Resources: The not existence and the lack of tagged Arabic Spontaneous Dialogues and Instant Messages corpora for Egyptian Arabic corpus make turn segmentation task far more challenging. Since manual construction of tagged corpus is time-consuming and expensive [9], it is difficult to build large tagged corpus for Arabic dialogue acts. Therefore, the researchers had to build their own resources for testing their approaches. Consequently, we used JANA corpus, which a multi-genre corpus of Arabic dialogues labeled for Arabic Dialogues Language Understanding (ADLU) at utterance level ant it comprises spontaneous dialogues and IM for Egyptian dialect; it developed by $[10,11]$. JANA corpus has collected, segmented and annotated manually. JANA consists of approximately 3001 turns with average 6.7 words per turn, contains 4725 utterances with average 4.3

\footnotetext{
${ }^{3}$ Examples are written as Arabic (Buckwalter transliteration schema,
} English translation) words per utterance, and 20311 words. The list of dialogue acts "dialogue acts schema" which have been used in the annotation of utterances in JANA corpus is shown in Table 1 ; this schema developed by [12].

Table 1. Dialgoues Acts Annotation Schema

\begin{tabular}{lll}
\hline Request Acts & & Response Acts \\
\cline { 1 - 1 } Taking-Request & & Service-Answer \\
Service-Question & & Other-Answer \\
Confirm-Question & & Agree \\
YesNo-Question & & Disagree \\
Choice-Question & & Greeting \\
Other-Question & & Inform \\
Turn-Assign & & Thanking \\
Other Acts & & Apology \\
\hline Opening & MissUnderstandingSign \\
Closing & Correct \\
Self-Introduce & Pausing \\
& Suggest \\
& Promise \\
& Warning \\
& Offer \\
& &
\end{tabular}

\section{RELATED WORKS}

Webb and Hardy are noticing that there are two ways to understand the dialogues language [13]:

- Shallow understanding: It is simple spotting keywords, or having lists of, for example, every location recognized by the system. Several systems are able to decode directly from the acoustic signal into semantic concepts precisely because the speech recognizer already has access to this information.

- Deeper analysis: Using linguistic methods; including part-of-speech (POS) tagging, syntactic parsing and verb dependency relationships.

Using Machine Learning (ML) for solving the DA classification problem, researchers have not historically published the split of training and testing data used in their experiments, and in some cases methods to reduce the impact of the variations that can be observed when choosing data for training and testing have not been used [3]. Moreover, DAs are practically used in many live dialogue systems such as Airline Travel Information Systems (ATIS) [14], DARPA [15], and VERBMOBIL project [16]. N-gram models can be considered the simplest method of DA classification based on some limited sequence of previous DAs such as $[3,13,17,18]$ and sometimes used with Hidden Markova Model (HMM) such as [19]. In addition, there are other approaches such as Transformation-Based Learning (TBL)[20], and Naïve Bayesian [21]. These approaches are tested and designed for non-Arabic dialogues such as English, Germany, and France that completely differs for Arabic dialogues.

To the best of our knowledge, there are few works interested in Arabic dialogue acts classification such as [22] are used Naïve Bayes and Decision Trees. [23] are used utterances semantic labelling based on the frame grammar formalism. [24] are used syntactic parser context free grammar with HHM. [5] are Conditional Random Fields (CRF) to semantically label spoken Tunisian dialect turns. $[25,26]$ are used Arabic function words such as "هل" "do/does", "كيف" 
"How" to classify questions and non-questions utterances with Decision Tree classifier. These approaches designed and applied on MSA or Tunisian dialect. To the best of our knowledge, there is no published word for understanding Egyptian Arabic or Egyptian dialect. The survey by [27] presents background and the progress made in understanding Arabic dialogues.

In this work we complete the work of [28] which present the first steps for understanding the Egyptian Arabic when built the annotated corpus for Egyptian Arabic dialogues namely "JANA" and present the turns segmentation into utterances classifier.

\section{METHODOLOGY}

Support Vector Machine (SVM) is a supervised machine learning that has been shown to perform well on text classification tasks, where data is represented in a high dimensional space using sparse feature vectors $[29,30]$. Moreover, the SVM is robust to noise and the ability to deal with a large number of features effectively [31]. The SVM classifier is trained to discriminate between examples of each class, and those belonging to all other classes combined. During testing, the classifier scores on an example, are combined to predict its class label [32].

Most of works have dealt with the dialogues act classification problem for spoken dialogues using SVM; they usually used a semantic labelling. In this paper, we proposed a classifier based on multi-class SVM that reduces the running time, and reduce the training cost and time. For instance, if we have 24 dialogues acts, we needs 24 binary classifiers, but here we proposed a one classifier. We are working on the hypothesis that the dialogues act problem can solved as text-chunking problem.

The proposed approach "YOSR" is an SVM approach, which a Machine Learning based, involves a selected set of features, extracted from annotated utterances, that is used to generate a statistical model for utterance act prediction. We used YamCha SVM toolkit ${ }^{4}$ that converts utterances classification task to a text-chunking task.

There are three processes to do as preprocessing the input utterances before running the YOSR classifier. These processes are:

- Normalization: to avoid writing errors from the transcription, we normalized the transcribed turns (unified Arabic characters) as

○ Convert Hamza-under-Alif “!”, Hamza-over-Alif “l”, and Madda-over-Alif "I" to Alif ""”

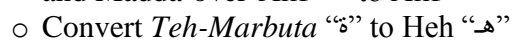

م Convert Alif-Maksura "ى" to Yeh "هي".

- Split "و" ( $w$, and) from the original words: Sometimes the writers write the conjunction " $g$ " ( $w$, and) concatenated to the next word. For instant, "وقال" (wqAl, and he talked) the original word "قال" ( $q A l$, he talked) is concatenated with the conjunction " $g$ " ( $w$, and). Detect and split " $g$ " ( $w$, and) from the original words has been done using Wawanizer Toolkit [10].

- The utterances are transliterated from Arabic to Latin based ASCII characters using the Buckwalter transliteration scheme $^{5}$.

\footnotetext{
${ }_{5}^{4}$ Available at http://chasen.org/ taku/software/yamcha/

${ }^{5}$ http://languagelog.ldc.upenn.edu/myl/ldc/morph/buckwalter.html
}

There are two phases has employed for carrying out the classification task in our approach, training phase and test phase. The training phase generates the classification model using a set of classification features. In the test phase, the classification model is utilized to predict a class for each token (word). In the training phase, each word is represented by a set of features and its actual DA tag in order to produce an SVM model that is able to predict the dialogue act of the utterance.

We used the BIO format (Beginning $B$ of the ACT, Inside $I$ the ACT, and Outside $O$ the ACT) or sometimes IOB Format, which developed for text-chunking by [33]. For instance, each word in utterances that refer to dialogue act "ServiceQuestion" will be represented as the first word of utterance will tagged by "B-Service_Question" and the other words will tagged by "I-Service_Question".

The first step in our approach is to extract the significant features from the training data. Consequently, we study the impact of the features individually by using only one feature at a time and measure the classifier's performance using the F-measure metric. Finally, according to the performance achieved, we select the optimized features for the proposed approach "YOSR".

\subsection{Features Selection}

Feature selection refers to the task of identifying a useful subset of features chosen to represent elements of a larger set.

Contextual word: The features of a sliding window, including a word n-gram that includes the candidate word, along with previous and following words. For instance, in the training corpus the word "عايز" (EAyz, want) appears frequently before a user's request that indicate a Request act or Service-Question act. Therefore, the classifier will use this information to predict a Service-Question act for this utterance.

Morphological Features: We used word Part-Od-Speech (POS); the sequence of NOUN and PROPER NOUN indicates the speaker needs to introduce himself or his company, for example "مصر للطيران"(mSr llTyrAn, Egypt Air). In addition, the sequence of preposition, NOUN or PROPER NOUN indicates the speaker needs to greet or return greet the other one. The consequence of NOUN and PROPER NOUN indicate the speaker needs to introduce himself or his company, for example "و عليكم السلام" (wElykm AlslAm, Peace be upon you).

Utterances Features: Using of the utterances meta information can help dialogue act classification process [3436]. Also, Knowing what happened before current utterance can help the classification task $[37,38]$. We used:

- Utterance Speaker Type: the speaker type Operator or Customer of the current utterance can help to determine the act of utterance. For instance, the act "Service-Question" is related to the customer because he connected to service support service to asking about a provided service, but the act "Other-Question" and "Choice-Question" is related to operator because the operator asking the client for his name or choosing the client to select one of the provided services.

- Previous Utterance Act: Knowing the previous utterances acts sequence in the dialogue help the classifier to predict the act of current utterance. For instance, the act "Agree" and "Disagree" is almost followed by the "ConfirmQuestion" act. 


\section{EXPERIMENTAL SETUP AND RESULTS}

In order to measure the effect of complexity of each dialogues domain (Banks, Flights, and Mobile Network Operators) on classification accuracy, we experiment on each dialogue domain separately and one experiment to overall combined data. We split each domain based on dialogue turn boundary into $70 \%$ training dataset, $20 \%$ development dataset (DEV), and the $10 \%$ test dataset as shown in Table 2 . The results are reported using standard metrics of Accuracy (Acc), Precision $(\mathrm{P})$, Recall $(\mathrm{R})$, and the F1 score $\left(\mathrm{F}_{1}\right)$.

$$
F_{1}=\frac{2 P R}{P+R}
$$

Table 2. Corpus training, development (DEV) and test datasets

\begin{tabular}{|c|c|c|c|c|c|}
\hline & Domain & Datasets & Dialogues & Turns & Utterances \\
\hline \multirow{2}{*}{$\begin{array}{l}\text { D̃ } \\
\text { o } \\
\text { की }\end{array}$} & Banks & $\begin{array}{l}\text { DEV } \\
\text { Test } \\
\text { Training }\end{array}$ & $\begin{array}{l}4 \\
5 \\
17\end{array}$ & $\begin{array}{l}115 \\
226 \\
782\end{array}$ & $\begin{array}{l}193 \\
368 \\
1,234\end{array}$ \\
\hline & Flights & $\begin{array}{l}\text { DEV } \\
\text { Test } \\
\text { Training }\end{array}$ & $\begin{array}{l}5 \\
7 \\
14\end{array}$ & $\begin{array}{l}145 \\
224 \\
773\end{array}$ & $\begin{array}{l}242 \\
364 \\
1,186\end{array}$ \\
\hline$\sum$ & $\begin{array}{l}\text { Mobile } \\
\text { Network } \\
\text { Operators }\end{array}$ & $\begin{array}{l}\text { DEV } \\
\text { Test } \\
\text { Training }\end{array}$ & $\begin{array}{l}3 \\
5 \\
22 \\
\end{array}$ & $\begin{array}{l}75 \\
197 \\
464 \\
\end{array}$ & $\begin{array}{l}109 \\
272 \\
757 \\
\end{array}$ \\
\hline \multicolumn{3}{|c|}{ Total } & 82 & 3,001 & 4,725 \\
\hline
\end{tabular}

In the training stage, the training is applied on the training dataset using selected features set and the results are analyzed to determine the best features set. The development stage is performed using the DEV dataset to define the best feature set which used in the test stage. In the test stage, the classifier is applied on the test dataset and the results are reported and discussed.

Table 3. The results of Banks test set

\begin{tabular}{|l|l|l|l|}
\hline \multicolumn{1}{|c|}{ Act } & Precision & Recall & F $_{\mathbf{1}}$ \\
\hline Agree & 90.57 & 97.96 & 94.12 \\
\hline Closing & 100 & 100 & 100 \\
\hline Confirm_Question & 32.56 & 45.16 & 37.84 \\
\hline Disagree & 100 & 60 & 75 \\
\hline Greeting & 92.86 & 96.3 & 94.55 \\
\hline MissUnderstandingSign & 60 & 75 & 66.67 \\
\hline Other_Answer & 46.15 & 40 & 42.86 \\
\hline Other_Question & 71.43 & 38.46 & 50 \\
\hline Pausing & 50 & 25 & 33.33 \\
\hline SelfIntroduce & 100 & 30 & 46.15 \\
\hline Service_Answer & 69.07 & 81.71 & 74.86 \\
\hline Service_Question & 78.26 & 56.25 & 65.45 \\
\hline Taking_Request & 100 & 100 & 100 \\
\hline Thanking & 69.23 & 81.82 & 75 \\
\hline Turn_Assgin & 72.73 & 100 & 84.21 \\
\hline \multicolumn{1}{|c|}{ Over All } & $\mathbf{7 2 . 7 5}$ & $\mathbf{7 2 . 7 5}$ & $\mathbf{7 2 . 7 5}$ \\
\hline
\end{tabular}

We classify utterances labelling task as a Multi-classification problem. Therefore, the proposed approach is tested using PAIRWISE and ONE vs ALL (OVA) approaches and we found the ONE vs ALL approach achieves the best performance in this task. Moreover, the selected features are tested on window size within ranges from $-1 /+1$ to $-5 /+5$. We found that a context size of $-2 /+2$ achieves the best performance.
Table 4. The results of Flights test set

\begin{tabular}{|c|c|c|c|}
\hline Act & Precision & Recall & F1 \\
\hline Agree & 93.42 & 84.52 & 88.75 \\
\hline Closing & 100 & 100 & 100 \\
\hline Confirm_Question & 44.44 & 26.09 & 32.88 \\
\hline Disagree & 33.33 & 18.18 & 23.53 \\
\hline Greeting & 90.91 & 88.24 & 89.55 \\
\hline Other_Answer & 29.17 & 58.33 & 38.89 \\
\hline Other_Question & 37.5 & 50 & 42.86 \\
\hline Pausing & 25 & 16.67 & 20 \\
\hline SelfIntroduce & 92.86 & 100 & 96.3 \\
\hline Service_Answer & 59.77 & 71.23 & 65 \\
\hline Service_Question & 48.89 & 64.71 & 55.7 \\
\hline Thanking & 70 & 63.64 & 66.67 \\
\hline Turn_Assgin & 66.67 & 57.14 & 61.54 \\
\hline Over All & 65 & 64.11 & 64.55 \\
\hline
\end{tabular}

Table 5. The results of Mobile Network Operators test set

\begin{tabular}{|l|l|l|l|}
\hline \multicolumn{1}{|c|}{ Act } & Precision & Recall & F1 \\
\hline Agree & 65.52 & 63.33 & 64.41 \\
\hline Apology & 100 & 25 & 40 \\
\hline Confirm_Question & 66.67 & 58.33 & 62.22 \\
\hline Disagree & 50 & 60 & 54.55 \\
\hline Greeting & 91.3 & 84 & 87.5 \\
\hline Other_Question & 81.82 & 90 & 85.71 \\
\hline Pausing & 100 & 63.64 & 77.78 \\
\hline SelfIntroduce & 100 & 76.92 & 86.96 \\
\hline Service_Answer & 64.29 & 88.52 & 74.48 \\
\hline Service_Question & 58.33 & 80 & 67.47 \\
\hline Thanking & 90.91 & 83.33 & 86.96 \\
\hline Turn_Assgin & 60 & 81.82 & 69.23 \\
\hline \multicolumn{1}{|c|}{ Over All } & $\mathbf{6 8 . 0 1}$ & $\mathbf{6 8 . 2 7}$ & $\mathbf{6 8 . 1 4}$ \\
\hline
\end{tabular}

Table 3, Table 4 and Table 5 shows the results for each domain Banks, Flights, and Mobile Networks Operators respectively and Table 6 shows the results of overall combined data experiment. The results show that Flights dialogues have $F_{1}$ fewer than the other domains. Moreover, the analysis of error results shows that the errors occur due to one of these reasons:

- There are some sentences/words can refers to more than meaning "dialogue acts" such as:

○ "شكر" (\$krA, Thank you) usually it means "thanks" (thank you) but sometimes it means "disagree" (No) أي استفسار ثناني " when comes after utterance such as " "حضرتك (> y AstfsAr tAny HDrtk, Any other services)

○" “عو " (EfwA, you are welcome) usually it means "you are welcome" as a reply of "thank you" statement, but sometimes it means "miss understanding sign" (sorry).

- Some utterance needs to add semantic features to the classifier "deeply semantic analysis". For instance, for the ولكن طبعا لازم يكون عدي عليها 6 " " 6 " شهور" (wlkn TbEA lAzm ykwn Edy ElyhA 6 \$hwr, Make sure you must get it since 6 months). The customer has responded utterance such as لا لا هي عدي عليها 4 سنين. (lA lA hy Edy ElyhA 4 snyn, No it since 4 years). The proposed classifier is classified the customer utterance as "disagree 
act" because it contains "y" (lA, No) in spite of the customer agreement with the operator warning.

- The instant messages utterances (Mobile Network Operators) are contained many of writing errors that confused the classifier

Table 6. The results of Overall combined test set

\begin{tabular}{|c|c|c|c|}
\hline Act & Precision & Recall & F1 \\
\hline Agree & 86.94 & 91.04 & 88.94 \\
\hline Apology & 100 & 50 & 66.67 \\
\hline Closing & 100 & 100 & 100 \\
\hline Confirm_Question & 40 & 33.66 & 36.56 \\
\hline Disagree & 76.47 & 50 & 60.47 \\
\hline Greeting & 91.57 & 88.37 & 89.94 \\
\hline MissUnderstandingSign & 100 & 20 & 33.33 \\
\hline Other_Answer & 39.02 & 47.06 & 42.67 \\
\hline Other_Question & 58.33 & 60 & 59.15 \\
\hline Pausing & 71.43 & 47.62 & 57.14 \\
\hline SelfIntroduce & 96.43 & 75 & 84.37 \\
\hline Service_Answer & 67.44 & 80.56 & 73.42 \\
\hline Service_Question & 60.83 & 72.28 & 66.06 \\
\hline Taking_Request & 100 & 75 & 85.71 \\
\hline Thanking & 77.14 & 79.41 & 78.26 \\
\hline Turn_Assgin & 76.67 & 88.46 & 82.14 \\
\hline Over All & 70.61 & 70.12 & 70.36 \\
\hline
\end{tabular}

Moreover, the results show that YOSR classifier yields a good performance and efficiency in understanding Arabic Egyptian dialect dialogues for all domains without using any special lexicons, cues, or rules for each domain.

\section{CONCLUSION}

In this paper, we present a ML approach using SVM to solve the problem of automatic understanding of the Arabic dialogues task for Egyptian dialect at the utterance level; namely, YOSR. The proposed classifier has tested on corpus consists of spontaneous dialogues and IM for Egyptian dialect.

The results obtained that YOSR classifier is very promising. To the best of our knowledge, these are the first results reported for understanding the Egyptian dialect.

As perspectives, we plan to improve YOSR by adding a general cues for call-centers domain, morphological features such as the first verb type and Lemma, context-based features, and dialect words treatments. Moreover, we intend to extend the training corpus "JANA" to improve the classification results.

\section{REFERENCES}

[1] Elmadany, A.A., Abdou, S.M., Gheith, M.: Recent Approaches to Arabic Dialogue Acts Classifications. 4th International Conferences on Natural Language Processing (NLP-2015) - Computer Science \& Information Technology (CS \& IT) Series 5, 117-129 (2015)

[2] Stolcke, A., Ries, K., Coccaro, N., Shriberg, E., Bates, R., Jurafsky, D., Taylor, P., Martin, R., Ess-Dykema, C.V., Meteer, M.: Dialogue Act Modeling for Automatic Tagging and Recognition of Conversational Speech. Computational Linguistics 26, 339-373 (2000)
[3] Webb, N.: Cue-Based Dialogue Act Classification. Department of Computer Science. University of Sheffield, England (2010)

[4] Traum, D., Heeman, P.A.: Utterance units in spoken dialogue. Dialogue processing in spoken language systems, pp. 125-140. Springer, Berlin Heidelberg (1997)

[5] Graja, M., Jaoua, M., Belguith, L.H.: Discriminative Framework for Spoken Tunisian Dialect Understanding. 2nd International Conference on Statistical Language and Speech Processing, SLSP 2014, pp. 102-110. (2013)

[6] Pasha, A., Al-Badrashiny, M., Diab, M., Kholy, A.E., Eskander, R., Habash, N., Pooleery, M., Rambow, O., Roth, R.M.: MADAMIRA: A Fast, Comprehensive Tool for Morphological Analysis and Disambiguation of Arabic. Language Resources and Evaluation Conference (LREC 2014), pp., (2014)

[7] Elmahdy, M., Rainer, G., Wolfgang, M., Slim, A.: Survey on common Arabic language forms from a speech recognition point of view. In proceeding of International conference on Acoustics (NAG-DAGA), pp. 63-66. (2009)

[8] Zaidan, O.F., Callison-Burch, C.: Arabic dialect identification. Computational Linguistics 52, (2012)

[9] Zaghouani, W.: Critical Survey of the Freely Available Arabic Corpora. In proceeding of Workshop on Free/Open-Source Arabic Corpora and Corpora Processing Tools (LREC2014). (2014)

[10] Elmadany, A.A., Abdou, S.M., Gheith, M.: Turn Segmentation into Utterances for Arabic Spontaneous Dialogues and Instant Messages. International Journal on Natural Language Computing (IJNLC) 4, 111-123 (2015)

[11] Elmadany, A.A., Abdou, S.M., Gheith, M.: JANA: An Arabic Human-Human Dialogues Corpus. IEEE $2^{\text {nd }}$ International Conference on Recent Trends in Information Systems (ReTIS), pp. IEEE, (2015)

[12] Elmadany, A.A., Abdou, S.M., Gheith, M.: Arabic Inquiry-Answer Dialogue Acts Annotation Schema. IOSR Journal of Engineering (IOSRJEN) 04, 32-36 (2014)

[13] Webb, N., Hardy, H.: Data-Driven Language Understanding for Spoken Language Dialogue. American Association for Artificial (2005)

[14] Seneff, S., Hirschman, L., Zue, V.W.: Interactive Problem Solving and Dialogue in the ATIS Domain. In proceeding of HLT '91: Proceedings of the Workshop on Speech and Natural Language, pp. 354-359. (1991)

[15] Pellom, B., Ward, W., Hansen, J., Cole, R., Hacioglu, K., Zhang, J., Yu, X., Pradhan, S.: University of Colorado Dialog Systems for Travel and Navigation. In proceeding of HLT '01: Proceedings of the First International Conference on Human Language Technology Research. (2001)

[16] Wahlster, W.: Verbmobil: Foundations of Speech-ToSpeech Translation. Springer (2000) 
[17] Hardy, H., Strzalkowski, T., Wu, M., Ursu, C., Webb, N., Biermann, A., Inouye, R., McKenzie, A.: Datadriven strategies for an automated dialogue system. In proceeding of the 42nd Annual Meeting on Association for Computational Linguistics. (2004)

[18] Webb, N., Hepple, M., Wilks, Y.: Dialogue Act Classification Based on Intra-Utterance Features. In proceeding of the AAAI Work-shop on Spoken Language Understanding. (2005)

[19] Boyer, K., Ha, E., Pillips, R., Wallis, M., Vouk, M., Laster, J.: Dialogue Act Modleing in a Complex TaskOrinted Domain. In proceeding of SIGDIAL, pp. $297-$ 305. (2010)

[20] Samuel, K., Carberry, S., Vijay-Shanker, K.: Dialogue act tagging with transformation-based learning. In proceeding of the 36th Annual Meeting of the Association for Computational Linguistics and 17th International Conference on Computational Linguistics. (1998)

[21] Grau, S., Sanchis, E., Castro, M., Vilar, D.: Dialogue Act Classification using a Bayesian Approach. In proceeding of 9th Conference Speech and Computer. (2004)

[22] Shala, L., Rus, V., Graesser, A.: Automatic Speech Act Classification In Arabic. In proceeding of Subjetividad y Procesos Cognitivos Conference pp. 284-292. (2010)

[23] Bahou, Y., Belguith, L.H., Hamadou, A.B.: Towards a Human-Machine Spoken Dialogue in Arabic. In proceeding of Workshop on HLT \& NLP within the Arabic world: Arabic Language and local languages processing: Status Updates and Prospects, at the 6th Language Resources and Evaluation Conference (LREC'08). (2008)

[24] Lhioui, C., Zouaghi, A., Zrigui, M.: A Combined Method Based on Stochastic and Linguistic Paradigm for the Understanding of Arabic Spontaneous Utterances. In proceeding of CICLing 2013, Computational Linguistics and Intelligent Text Processing Lecture Notes in Computer Science, pp. 549-558. Springer-Verlag Berlin Heidelberg, (2013)

[25] Hijjawi, M., Bandar, Z., Crockett, K.: User's Utterance Classification Using Machine Learning for Arabic Conversational Agents. In proceeding of 5th International Conference on Computer Science and Information Technology (CSIT), pp. 223-232. IEEE, (2013)

[26] Hijjawi, M., Bandar, Z., Crockett, K., Mclean, D.: ArabChat: an Arabic Conversational Agent. In proceeding of 6th International Conference on Computer Science and Information Technology (CSIT), pp. 227-237. IEEE Computer Society, (2014)

[27] Elmadany, A.A., Abdou, S.M., Gheith, M.: A Survey of Arabic Dialogues Understanding for Spontaneous Dialogues and Instant Message. International Journal on Natural Language Computing (IJNLC) 4, 75-94 (2015)

[28] Kudo, T., Matsumoto, Y.: Chunking with support vector machines. In proceeding of NAACL-01. (2001)

[29] Kudo, Y., Matsumoto, Y.: Use of Support Vector Learning for Chunk Identification In proceeding of CoNLL, pp. 142-144. (2000)

[30] Meselhi, M.A., Bakr, H.M.A., Ziedan, I., Shaalan, K.: A Novel Hybrid Approach to Arabic Named Entity Recognition. The 10th China Workshop on Machine Translation (CWMT 2014), pp. 93-103. (2014)

[31] Pradhan, S., Hacioglu, K., Krugler, V., Ward, W., Martin, J.H., Jurafsky, D.: Support vector learning for semantic argument classification. Machine Learning 60 Machine Learning, 11-39 (2005)

[32] Ramshaw, L.A., Marcus, M.P.: Text Chunking Using Transformation-based Learning. In proceeding of the Third ACL Workshop on Very Large Corpora (WVLC 1995). (1995)

[33] Kim, S.N., Cavedon, L., Baldwin, T.: Classifying dialogue acts in one-on-one live chats. In proceeding of the Conference on Empirical Methods in Natural Language Processing (EMNLP 2010), pp. 862-871. (2010)

[34] Ivanovic, E.: Automatic utterance segmentation in instant messaging dialogue. In proceeding of The Australasian Language Technology Workshop, pp. 241249. (2005)

[35] Ivanovic, E.: Automatic instant messaging dialogue using statistical models and dialogue acts. Faculty of Engineering, Computer Science and Software Engineering. University of Melbourne (2008)

[36] Sridhara, V.K.R., Bangaloreb, S., Narayanana, S.: Combining lexical, syntactic and prosodic cues for improved online dialog act tagging. Computer Speech \& Language 23, 407-422 (2009)

[37] Eugenio, B.D., Xie, Z., Serafin, R.: Dialogue act classification, higher order dialogue structure, and instance-based learning. Dialogue and Discourse 1, 124 (2010) 